\title{
COMMUTATIVITY OF ENDOMORPHISM RINGS OF IDEALS. II ${ }^{1}$
}

\author{
S. ALAMELU
}

\begin{abstract}
Let $R$ be a commutative ring. In (1), it was proved that a ring $R$ with noetherian total quotient ring is self-injective if and only if the endomorphism ring of every ideal is commutative. We prove here that if the ring is coherent and is its own total quotient ring, then $R$ is self-injective if and only if $\operatorname{Hom}(I, I)=R$ for every ideal $I$ of $R$.
\end{abstract}

In (1), we discussed the commutativity of endomorphism rings of ideals. There, we proved that a ring with noetherian total quotient ring is self-injective if and only if the endomorphism ring of every ideal is commutative. The generalization of this to nonnoetherian total quotient rings seems extremely difficult. We will call an element $x$ of $R$ a stable element if every nonzero homomorphism $(x)$ to $R$ is a multiplication by an element of $R$. We remark that if every element of $R$ is stable, then $\operatorname{Hom}(I, I)$ is commutative for every ideal $I$ of $R$. The converse is in general not true. But, we have some partial answers in this direction. If the ring is coherent and is its own total quotient ring and if $\operatorname{Hom}(I, I)=R$ for every ideal generated by 2 elements then every element of $R$ is stable. On the other hand we have an example of a noncoherent ring which is its own total quotient ring which has a nonstable element but $\operatorname{Hom}(I, I)$ is commutative for every ideal $I$ of $R$.

We start with an example of a ring which is not self-injective, but $\operatorname{Hom}(I, I)$ is commutative for every ideal $I$ of $R$.

EXAMPLE. $R=K\left[x_{1}, x_{2}, \ldots\right] /\left(x_{1}^{2}, x_{2}^{2}, \ldots\right)$ where $x_{1}, x_{2}, \ldots$ are indeterminates. $R$ is the inductive limit of the rings $K\left[x_{1}, x_{2}, \ldots, x_{n}\right] /\left(x_{1}^{2}, \ldots, x_{n}^{2}\right), n$ $=1,2, \ldots$, and each of them is self-injective. It follows that $\operatorname{Hom}(I, I)$ is commutative for every ideal $I$. It is easy to see that $R$ is not self-injective.

We note that in this example $R$ has the following properties. Every element of $R$ is stable, $\operatorname{Hom}(I, I)=R$ for every finitely generated ideal $I$. $R$ is further quasi-local and the zero ideal of $R$ is irreducible. We will presently see that these are not isolated phenomena.

We note first the following general result.

Proposition 1. If every element of $R$ is stable, then $\operatorname{Hom}(I, I)$ is commutative for every ideal $I$ of $R$.

Received by the editors May 30, 1975.

AMS (MOS) subject classifications (1970). Primary 13C05; Secondary 13A99.

Key words and phrases. Total quotient ring, coherent ring, self-injective ring, irreducible ideal.

1 Most of the results of this paper are contained in the author's doctoral dissertation accepted by the Madurai University. The author wishes to thank her advisor Dr. K. R. Nagarajan for his guidance and encouragement.

(1) American Mathematical Society 1976 
Proof. It is enough if we prove that for any 2 elements $f, g \in \operatorname{Hom}(I, I)$ and $x \in I, f g(x)=g f(x)$. But this follows immediately: for, as $x$ being stable, $f(x)$ and $g(x)$ are multiples of $x$.

Proposition 2. Let $R$ be a quasi-local ring (a ring with unique maximal ideal). Suppose $\operatorname{Hom}(I, I)=R$ for every ideal I generated by two elements, then $(0)$ is irreducible.

Proof. Suppose (0) is reducible, then $(0)=Q_{1} \cap Q_{2}$. Take $0 \neq x \in Q_{1}$, $0 \neq y \in Q_{2}$. Let $F$ be the homomorphism which takes $x$ to $x$ and $y$ to 0 and $a x+b y$ to $a x$. This is a well-defined homomorphism from $(x, y)$ to $(x, y)$. By hypothesis, $F$ is a multiplication by an element of $R$. Since $R$ has no nontrivial idempotents and $F^{2}=F$, we get a contradiction.

DEFINITION. $R$ is said to be coherent if every finitely generated ideal of $R$ is finitely related.

Proposition 3. Let $R$ be a coherent quasi-local ring which is its own total quotient ring.

(1) Suppose $\operatorname{Hom}(I, I)=R$ for every ideal I generated by two elements. Then every element of $R$ is stable and hence $\operatorname{Hom}(I, I)$ is commutative for every ideal $I$ of $R$.

(2) $\operatorname{Hom}(I, I)=R$ for every ideal $I$ if and only if $R$ is self-injective.

Proof. By Proposition 2, (0) is irreducible. An element $a$ of $R$ is stable if and only if whenever $b \notin(a)$, Ann $(a) \nsubseteq$ Ann $b$.

Consider $x$ and $y$ any two elements of $R$ and assume $y$ is not a multiple of $x$. Consider the relation module $K$ of $(x, y) . K=\{(s, t) \mid s x+t y=0\}$. Since $R$ is coherent, $K$ is finitely generated, generated by $\left(s_{i}, t_{i}\right), i=1,2, \ldots, n$. Since $y$ is not a multiple of $x$, it follows that $I=\left(t_{1}, t_{2}, \ldots, t_{n}\right)$ is a proper ideal.

Now Ann $I \supset \operatorname{Ann}\left(t_{1}\right) \cap \operatorname{Ann}\left(t_{2}\right) \cap \cdots \cap \operatorname{Ann}\left(t_{n}\right)$ and this is $\neq 0$ since (0) is irreducible and Ann $\left(t_{i}\right)=0$ implies $t_{i}$ is a unit. Hence Ann $I \cap(x)$ $\neq(0)$. Let $t \in R$ be such that $t x \in$ Ann $I$. Consider the mapping $f: x \mapsto 0$, $y \mapsto t x$, and extend by linearity. This is well defined, for, if $m x+n y=0$, then $n \in I$, hence $n t x=0$. By hypothesis, $f:(x, y) \rightarrow(x, y)$ is multiplication by an element of $R$. I.e., there exists $s \in R$ such that $s y=t x \neq 0$ and $s x=0$. Thus every element of $R$ is stable.

(2) Let $f: J \rightarrow R$ be any homomorphism. Since each element $x \in J$ is mapped into a multiple of $x, f$ is actually a map from $J$ to $J$. Hence $f$ is a multiplication by an element of $R$. Hence $R$ is self-injective. The converse is obvious.

The result is also true globally by the following

Proposition 4. Let $R$ be a coherent ring which is its own total quotient ring.

(1) Suppose $\operatorname{Hom}(I, I)=R$ for every ideal I generated by two elements. Then every element of $R$ is stable and hence $\operatorname{Hom}(I, I)$ is commutative for every ideal $I$ of $R$.

(2) $\operatorname{Hom}(I, I)=R$ for every ideal $I$, if and only if $R$ is self-injective.

Proor. (1) Let $x, y$ be any two elements of $R$. Let $I=(x, y)$. Since $I$ is finitely related, $\operatorname{Hom}(I, I)_{P} \approx \operatorname{Hom}\left(I_{P}, I_{P}\right), \forall P \in \operatorname{Spec} R$. By Proposition 3 , $x$ is mapped into a multiple of $x$ at each localisation. Let $f \in \operatorname{Hom}((x), R)$. 
For every $P \in \operatorname{Spec} R$, there exists $s \in R-P$ such that $f(x) s=t x$. The ideal generated by $s$ for various primes is $R$, hence $f(x)$ is a multiplication by an element of $R$. Hence the element $x$ is stable.

(2) Once again if $J$ is any ideal of $R$ any map $J \rightarrow R$ is actually a map $J \rightarrow J$ and therefore is a multiplication by an element of $R$.

The converse is obvious.

If $R$ is noetherian with total quotient ring $Q$, we have seen that $Q$ is selfinjective and therefore each element $x \in Q$ is stable, if $\operatorname{Hom}_{R}(I, I)$ is commutative for all ideals. Proposition 4 says that a similar result is true for coherent $R$ if we further assume $\operatorname{Hom}_{Q}(I, I)=Q$ for all ideals $I$ of $Q$. The question is whether we can relax this condition to saying $\operatorname{Hom}(I, I)$ is commutative. At present, we do not have any example of a coherent ring which is its own total quotient $\operatorname{ring}, \operatorname{Hom}_{R}(I, I)$ is commutative for every ideal $I$ of $R$, and $R$ has a nonstable element. But it seems that at least coherence is a necessary condition for stableness. Our next example is a noncoherent ring which has a nonstable element, and $\operatorname{Hom}_{R}(I, I)$ is commutative for each ideal $I$. The idea of the example is motivated by the following general result.

Suppose $\left\{A_{i}\right\}, i \in I$, is a family of rings, each of which has the property that the endomorphism rings of ideals are commutative. Suppose for every $i$, Ann $A_{i}=0$. Then $\oplus A_{i}$ also has the property, that the endomorphism rings of ideals are commutative. The proof of this is not essentially different from the case of finite families.

Lemma. Let $A$ and $B$ be two rings (not necessarily with identity) such that Ann $A=$ Ann $B=0$. Suppose for every ideal $I$ in $A$ or $B, \operatorname{Hom}(I, I)$ is commutative. Let $C=A \oplus B$. Then $C$ has the property that $\operatorname{Hom}(I, I)$ is commutative for every ideal $I$ in $C$.

Proof. Let $I$ be an ideal in $A \oplus B$. Let $P_{1}$ and $P_{2}$ be projection on $A$ and $B$. Let $I_{i}=P_{i} I, i=1,2$. Define a map $f_{i}$ from $I_{i}$ to $I_{i}, i=1,2$ as follows. Let $a \in I_{1}$ and $x \in I_{2} . f_{1}(a)=P_{1} f(a, b)$ and $f_{2}(x)=P_{2} f(y, x)$.

Claim. $f_{i}, i=1,2$,are well defined. We show that if $(a, b)$ and $\left(a, b^{\prime}\right) \in I$, $P_{1} f(a, b)=P_{1} f\left(a, b^{\prime}\right)$. Let $f(a, b)=\left(a_{1}, \alpha\right)$, and $f\left(a, b^{\prime}\right)=\left(a^{\prime}, \alpha^{\prime}\right)$. Then for any element $t \in A, t\left(a_{1}-a^{\prime}\right)=0$, i.e., $a_{1}=a^{\prime}$. Hence $f_{1}$ is well defined. Similarly $f_{2}$ is also well defined. $f_{1}$ and $f_{2}$ are clearly homomorphisms since $f$ is a homomorphism and $P_{i}$ are additive.

To show $\operatorname{Hom}(I, I)$ is commutative, it is enough to prove that for any element $(a, b) \in I$, and any two elements $f, g \in \operatorname{Hom}(I, I) f g(a, b)=g f(a, b)$. Now

$$
f g(a, b)=f\left(g_{1}(a), g_{2}(b)\right)=\left(f_{1} g_{1}(a), f_{2} g_{2}(b)\right)=\left(g_{1} f_{1}(a), g_{2} f_{2}(b)\right)=g f(a, b) .
$$

EXAMPLE. Let $R$ be the total quotient ring of the ring

$$
K\left[x, y_{1}, y_{2}, \ldots\right] /\left(y_{1}^{2}, y_{2}^{2}, \ldots, x y_{1}, x y_{2}, \ldots\right) .
$$

Then $\operatorname{Hom}_{R}(I, I)$ is commutative for every ideal $I$ of $R$ and $R$ has a nonstable element.

Proof. The maximal ideal $R^{\prime}$ of $R$ is the direct sum of the rings $A$ $=x k[x]_{(x)}$ and $B=Y k[Y] /\left(Y^{2}\right)$ where $Y=\left(y_{1}, y_{2}, \ldots\right)$ and $Y^{2}=\left(y_{1}^{2}\right.$, $\left.y_{2}^{2}, \ldots\right)$. 
Ann $A=$ Ann $B=0$. Let $I$ be an ideal of $R$ and $f, g \in \operatorname{Hom}(I, I)$. Then $f_{i}$ and $g_{i}, i=1,2$, defined as in the Lemma are well defined homomorphisms. Since $A$ is an integral domain, $f_{1} g_{1}=g_{1} f_{1}$. As for $f_{2}$ and $g_{2}$, we notice that (since $f$ and $g$ are $R$-homomorphisms) they are in fact endomorphisms of $I_{2}$ considered as an ideal in $K[Y] / Y^{2}$, and hence commute. It is clear that $f g=g f$.

Denote by $x$ the coset of $x$. The homomorphism $x^{2} R \rightarrow R$ given by $x^{2} \mapsto x$ is not a multiplication by an element. Hence $x^{2}$ is a nonstable element.

REMARK. In the ring $k[x, y] /\left(x y, y^{2}\right)$, the ideal $(x, y)$ has noncommutative endomorphism ring. For example, take $f: x \mapsto x, y \mapsto 0$ and $g: x \mapsto y, y \mapsto 0$. One notices that the set of all zero divisors of the ring is the direct sum of $x k[x]$ and $y k[y] / y^{2}$ and $y$ annihilates the ring $y k[y] /\left(y^{2}\right)$. Thus, in Lemma, the hypothesis that Ann $A_{i}=0$ is necessary.

\section{REFERENCES}

1. S. Alamelu, On commutativity of endomorphism rings of ideals, Proc. Amer. Math. Soc. 37 (1973), 29-31. MR 47 \#213.

Department of Mathematics, Madurai University, Madurai 625 021, India

Current address: The Ramanujan Institute for Advanced Studies in Mathematics, Madras University, Madras-600 005, India 